\title{
Efficacy of radioiodine therapy for treating 20 patients with pulmonary metastases from differentiated thyroid cancer and a meta-analysis of the current literature
}

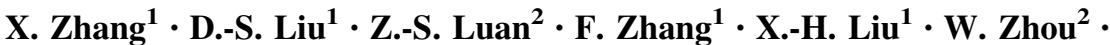 \\ S.-F. Zhong ${ }^{2} \cdot$ H. Lai ${ }^{3}$ (D)
}

Received: 3 June 2017/ Accepted: 30 October 2017/Published online: 8 November 2017

(c) The Author(s) 2017. This article is an open access publication

\begin{abstract}
Background Radioiodine therapy (RAI) after total or neartotal thyroidectomy is a recommended treatment for patients with pulmonary metastasis from differentiated thyroid cancer (DTC). However, the total effective rate of iodine-131 therapy remains controversial. This study aimed to determine the efficacy of RAI for treating patients with pulmonary metastasis from DTC, and to identify independent predictors of its efficacy.

Methods We conducted a retrospective study to evaluate 20 patients with pulmonary metastasis from DTC who underwent RAI at our center at first and performed a metaanalysis to evaluate relevant literature regarding the overall efficacy of RAI and subgroup-specific efficacies subsequently.

Results The efficacy rate at our center was $40 \%$, and no significant differences were observed according to sex, age, pathological type, metastasis state, or interval between the initial RAI and final surgery. The meta-analysis revealed that the pooled overall efficacy rate was $58 \%$, and significant differences were observed when we compared pulmonary metastasis versus pulmonary and other distant metastasis, age of $<40$ years versus age of $\geq 40$ years,
\end{abstract}

X. Zhang and D.-S. Liu contributed equally for this study.

H. Lai

laihong185@126.com

1 Department of Traditional Chinese Medicine, Qilu Hospital of Shandong University, Jinan, China

2 Department of Nuclear Medicine, The 88th Hospital of PLA, Tai' an, China

3 Department of Endocrinology, Qilu Hospital of Shandong University, 107 Wenhua Xi Road, Jinan 250012, People's Republic of China papillary thyroid cancer versus follicular thyroid cancer and male patients versus female patients.

Conclusions These results suggest that RAI is an effective treatment for patients with pulmonary metastasis from DTC after surgical treatment. The efficacy of RAI was significantly predicted by the presence of papillary thyroid cancer, age of $<40$ years, the absence of non-lung distant metastasis and female patients.

Keywords Differentiated thyroid cancer - Pulmonary metastasis · Radioiodine therapy $\cdot$ Efficacy $\cdot$ Meta-analysis

\section{Introduction}

Differentiated thyroid cancer (DTC) accounts for the majority of thyroid cancers, and includes papillary thyroid cancer (PTC) and follicular thyroid cancer (FTC) [1]. The lungs are the most common site of distant metastasis in patients with DTC [2-4]. Based on the 2009 guidelines from the American Thyroid Association, total or subtotal thyroidectomy followed by radioiodine therapy (RAI) is recommended for patients with pulmonary metastasis from DTC [5], and most studies regarding this treatment have revealed significant efficacy [6-9]. However, the overall efficacy of iodine-131 $\left({ }^{131} \mathrm{I}\right)$ therapy remains controversial. On the one hand, it is reported that the efficacy of RAI to DTC is associated with the expression of sodium iodide symporter (NIS). The presentation of NIS in DTC cells is responsible for radioiodine uptake. Some differentiated thyroid cancers have a poor prognosis for the iodine uptake is impaired [10]. On the other hand, most studies have used a retrospective design, did not use randomization, or did not control for the limitations of ethical principles and 
medical reality. Therefore, the present study retrospectively evaluated the clinicopathological characteristics and responses to ${ }^{131} \mathrm{I}$ therapy among 20 patients with pulmonary metastasis from DTC. Furthermore, we performed a meta-analysis of the current literature to identify factors that independently predicted RAI efficacy in cases of pulmonary metastasis from DTC.

\section{Methods}

\section{Patients}

Between 2005 and 2014, 20 patients with pulmonary metastases in DTC underwent RAI after undergoing surgical treatment at our Department of Nuclear Medicine (The 88th PLA Hospital). All the patients included in our retrospective study were consisted of 10 male patients and 10 female patients. The ages at the diagnosis ranged from 19 to 76 years (mean $45.7 \pm 17.6$ years), and 5 patients $(25 \%)$ experienced distant metastases to the bone. The pulmonary and bone metastases were mainly diagnosed based on pre- or posttreatment uptake of radioiodine that was identified during whole-body scanning (WBS). In cases where the WBS did not detect pulmonary metastasis, we also used computed tomography to screen for pulmonary nodes and tested for elevated serum thyroglobulin $(\mathrm{Tg})$ levels.

\section{Treatment}

The RAI was administered as a single $30-215 \mathrm{mCi}$ dose of ${ }^{131} \mathrm{I}$, with an intertreatment interval of $3-6$ months. All patients received no more than five treatments, and the results were evaluated using WBS and Tg measurements. At 3-5 days after each treatment, a quantitative WBS was performed using a dual-head gamma camera (GE Hawkeye SPECT or Symbia T2). Serum Tg measurements were performed using a chemiluminescence assay before each oral treatment.

\section{Criteria to evaluate treatment efficacy}

Complete remission (CR) was defined as a serum $\mathrm{Tg}$ level of $\leq 1.5 \mathrm{ng} / \mathrm{mL}$ and no clear ${ }^{131} \mathrm{I}$ uptake was detected during the WBS. Partial remission (PR) was defined as a serum $\mathrm{Tg}$ level of $\leq 1.5 \mathrm{ng} / \mathrm{mL}$ and no clear ${ }^{131}$ I uptake was detected during the WBS. The treatment was considered effective in cases with $\mathrm{CR}$ or $\mathrm{PR}$. Treatment ineffectiveness was defined as a serum $\mathrm{Tg}$ level of $>1.5 \mathrm{ng} / \mathrm{mL}$ and a clear ${ }^{131} \mathrm{I}$ uptake was detected during the WBS.

\section{Statistical analysis}

Continuous data were presented as mean \pm standard deviation, and categorical data were presented as number (\%). Differences in treatment response were compared using Fisher's exact test and IBM SPSS software (version 21.0; IBM Corp., Armonk, NY, USA). Differences were considered significant at $P$ values of $<0.05$.

\section{Meta-analysis}

We also performed a meta-analysis to evaluate the overall efficacy of RAI for patients with pulmonary metastasis from DTC, and to identify independent predictors of its efficacy. A search of the PubMed database was performed in October 2016 by two independent investigators using the following terms: "thyroid carcinoma OR thyroid neoplasm OR thyroid neoplasms OR thyroid cancer" AND" iodine OR radioiodine "AND" lung metastas* OR pulmonary metastas*". The inclusion criteria were: (1) the report was published in English (2) the study evaluated RAI for patients with pulmonary metastasis from DTC, and (3) the patients all underwent total or subtotal thyroidectomy before the RAI. The exclusion criteria were: (1) reviews, (2) studies with a small sample size ( $<15$ patients), and (3) unclear information regarding the outcomes and efficacy of RAI.

The meta-analysis was performed using the Cochrane Collaboration Review Manager (RevMan version 5.3). Pooled estimates of the effective rates were calculated using either a fixed-effects or random-effects model based on heterogeneity. Significant heterogeneity was considered present based on a Chi-square test $P$ value of $\leq 0.05$ or an $I^{2}$ value of $>50 \%$ [11]. The overall efficacy results were expressed as risk difference (RD) with the $95 \%$ confidence interval $(\mathrm{CI})$, and the results of the subgroup analyses were expressed as odds ratios (ORs) with $95 \%$ CIs. A funnel plot was used to test for the presence of publication bias.

\section{Results}

\section{Results of the RAI at our center}

The clinical characteristics of the 20 patients from our center are shown in Table 1. The patients received single doses of $30-215 \mathrm{mCi}$ and total doses of $249-895 \mathrm{mCi}$ (mean $559.0 \pm 143.1 \mathrm{mCi}$ ) during $2-5$ treatments (mean $4.5 \pm 0.9$ treatments). We observed CR in 5 patients (25\%) and PR in 3 patients (15\%), and the RAI was considered ineffective for the remaining 12 patients $(60 \%)$. Thus, the efficacy rate at our center was $40 \%$. We did not detect any statistically significant differences in efficacy according to 
Table 1 Clinical feature of the 20 patients with pulmonary metastases from DTC

\begin{tabular}{|c|c|c|c|c|}
\hline Clinical feature & No. of patients $(\%)$ & Effectiveness (CR/PR) & Ineffectiveness & $P$ \\
\hline \multicolumn{5}{|l|}{ Gender } \\
\hline Male & $10(50.0 \%)$ & 3 & 7 & \multirow[t]{2}{*}{0.650} \\
\hline Female & $10(50.0 \%)$ & 5 & 5 & \\
\hline \multicolumn{5}{|l|}{ Age (years) } \\
\hline$<40$ & $9(45.0 \%)$ & 5 & 4 & \multirow[t]{2}{*}{0.362} \\
\hline$\geq 40$ & $11(55.0 \%)$ & 3 & 8 & \\
\hline \multicolumn{5}{|l|}{ Pathological type } \\
\hline PTC & $14(70.0 \%)$ & 7 & 7 & \multirow[t]{2}{*}{0.325} \\
\hline FTC & $6(30.0 \%)$ & 1 & 5 & \\
\hline \multicolumn{5}{|l|}{ Metastasis state } \\
\hline PM & $5(25.0 \%)$ & 0 & 5 & \multirow[t]{2}{*}{0.055} \\
\hline PBM & $15(75.0 \%)$ & 8 & 7 & \\
\hline \multicolumn{5}{|c|}{ Interval between initial RAI and final surgery, months } \\
\hline$<5$ & $7(35.0 \%)$ & 1 & 6 & \multirow[t]{2}{*}{0.158} \\
\hline$\geq 5$ & $13(65.0 \%)$ & 7 & 6 & \\
\hline
\end{tabular}

$P M$ pulmonary metastases, $P B M$ pulmonary and bone metastases sex, age, pathological type, metastasis state, or interval between the initial RAI and the final surgery.

\section{Results of the meta-analysis}

Figure 1 shows that we identified 196 potentially relevant reports, although only 13 reports were included after

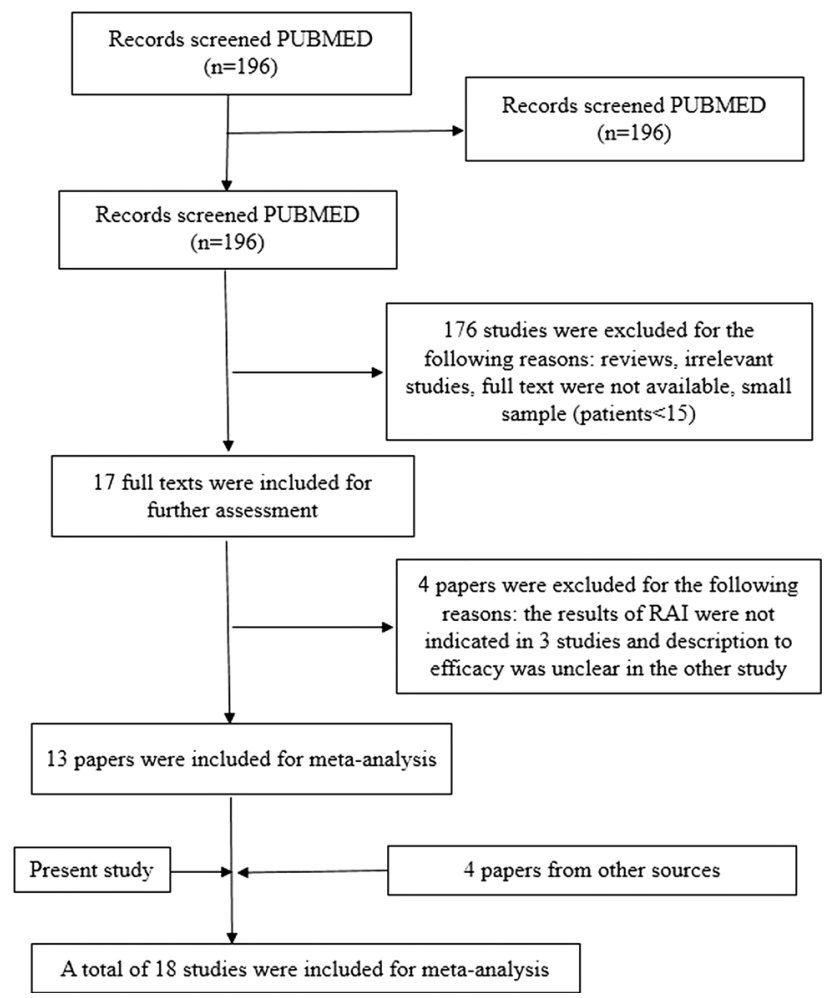

Fig. 1 The article selection procedure applying the inclusion and exclusion criteria. We also identified 4 reports from other sources that fulfilled the inclusion criteria. In addition to $\mathrm{CR}$ and PR, some studies defined effectiveness using cases with no evidence of disease, stable disease, or being alive with disease. The overall efficacy of RAI for pulmonary metastasis from DTC varied from 37.5 to $96.8 \%$ (Table 2). When we combined our study with the previous studies, we identified 18 studies with 1228 patients.

The pooled overall efficacy rate was $58 \%$ (RD 0.58 , 95\% CI 0.45-0.71, $P<0.00001$ ) (Fig. 2). Subgroup analyses revealed significant differences in the total efficacy rates when we compared pulmonary metastasis versus pulmonary and other distant metastasis (OR 7.29, 95\% CI 5.03-10.54, $P<0.00001$ ) (Fig. 3), age of $<40$ years versus age of $\geq 40$ years (OR 1.94, 95\% CI 1.09-3.46, $P=0.02$ ) (Fig. 4), and PTC versus FTC (OR 1.86, 95\% CI 1.10-3.15, $P=0.02$ ) (Fig. 5). No significant difference was observed when we compared the male and female patients (OR 1.12, 95\% CI 0.68-1.85, $P=0.65$ ) (Fig. 6).

We made a comparison between the results which correlated most closely to the results produced by us to give a better insight into the variation (with a total efficacy rate at 35-50\%). Significant differences were revealed in the total efficacy rate not only when we compared pulmonary metastasis versus pulmonary and other distant metastasis (OR 10.86, 95\% CI 5.12-23.00, $P<0.00001$ ) (Fig. 7) and PTC versus FTC (OR 4.63, 95\% CI 1.39-15.4, $P=0.01$ ) (Fig. 8), but also when we compared the male and female patients (OR 0.32, 95\% CI 0.12-0.85, $P=0.02$ ) (Fig. 9).

Figure 10 shows the funnel plot of the included studies, and does not indicate that significant publication bias is present. 
Table 2 Characteristics of included studies

\begin{tabular}{lll}
\hline Study & Definition of effectiveness & Total effective rate $(n)$ \\
\hline Bal et al. [6] & Ablated or partially ablated & $90.0 \%(18 / 20)$ \\
Biko et al. [17] & Complete biochemical remission or stable partial remission & $80.0 \%(16 / 20)$ \\
Durante et al. [3] & No radiological abnormalities or No persistent radioiodine uptake & $62.3 \%(190 / 305)$ \\
Hindié et al. [18] & Apparent remission or persistent disease or recurrent remission and relapse & $80.0 \%(16 / 20)$ \\
Ilgan et al. [13] & Complete response or stable disease & $40.5 \%(17 / 42)$ \\
Kalender et al. [12] & Complete response or partial response & $47.1 \%(8 / 17)$ \\
Küçük et al. [14] & Tg $<30$ ng/mL & $37.5 \%(12 / 32)$ \\
Lee et al. [19] & Alive with disease or no evidence of disease & $70.0 \%(14 / 20)$ \\
Long et al. [7] & Complete response or partial response & $95.2 \%(20 / 21)$ \\
Dottorini et al. [23] & 131 I lung uptake was no longer evident on the WBS and Tg continued to decrease & $75.0 \%(12 / 16)$ \\
Pace et al. [20] & Complete remission or partial remission or stable disease & $72.2 \%(13 / 18)$ \\
Pitoia et al. [15] & No evidence of disease or biochemical persistence & $45.8 \%(11 / 24)$ \\
Present study & Complete remission or partial remission & $40.0 \%(8 / 20)$ \\
Samuel et al. [8] & Complete response or partial response & $96.2 \%(25 / 26)$ \\
Schlumberger et al. [21] & Complete remission & $87.0 \%(20 / 23)$ \\
Schlumberger et al. [16] & Remission & $39.2 \%(112 / 286)$ \\
Song et al. [22] & Effective or stable & $82.4 \%(211 / 256)$ \\
Tachi et al. [9] & Partial remission or no change & $96.8 \%(60 / 62)$ \\
\hline
\end{tabular}

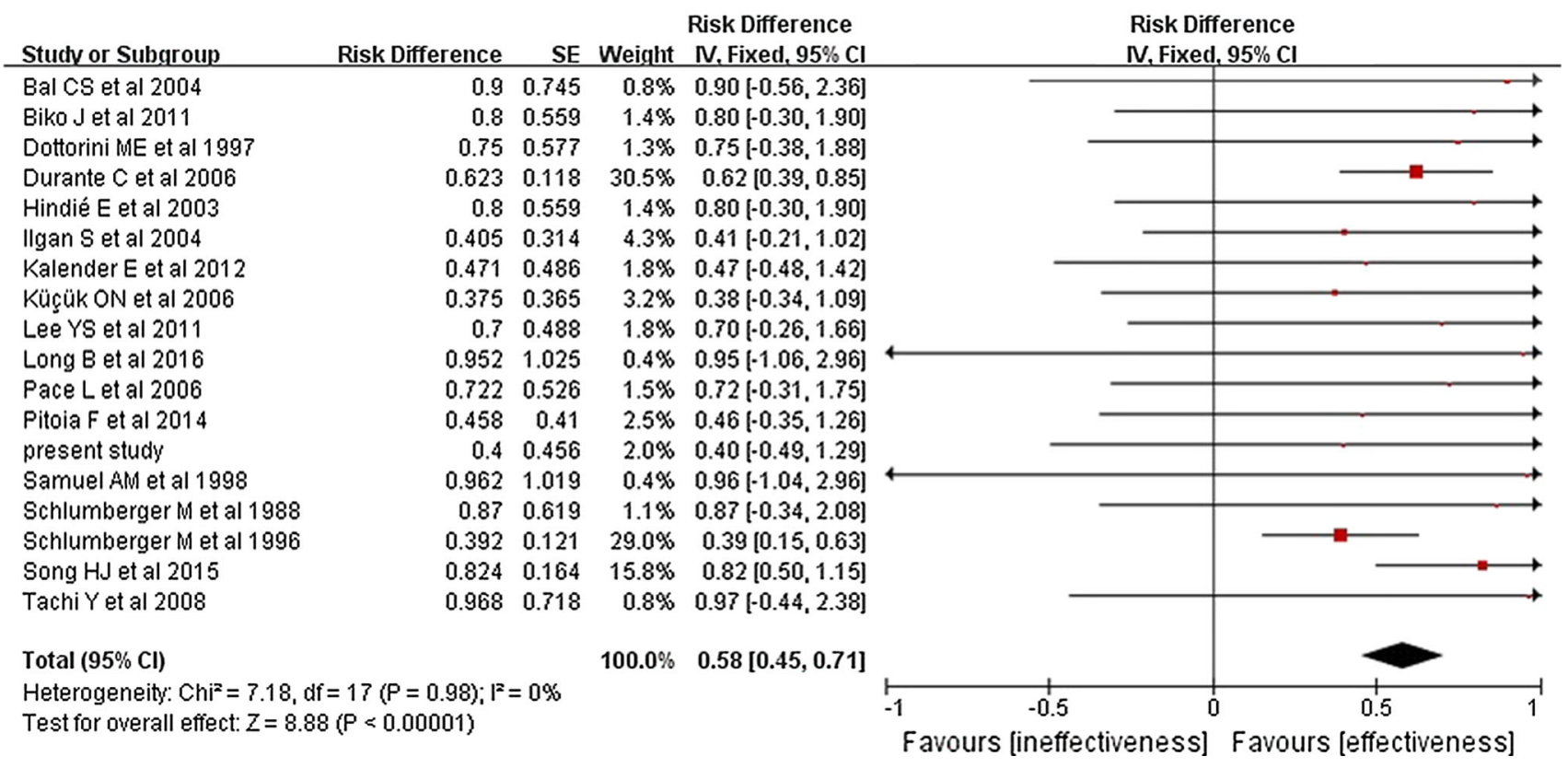

Fig. 2 Forest plots for the pooled overall efficacy rate using a fixed-effects model

\section{Discussion}

Numerous studies have reported the efficacy of RAI, which plays a key role in the treatment of patients with pulmonary metastasis from DTC. This retrospective study evaluated 20 patients who received RAI for pulmonary metastasis from DTC, and found that the efficacy rate was relatively low (40\%). Furthermore, none of the patients' characteristics were significantly associated with RAI efficacy. Therefore, we also performed a meta-analysis of related studies, and found that the overall efficacy rate was $58 \%$. To our knowledge, this is the first meta-analysis to evaluate the efficacy rate of RAI for pulmonary metastasis from DTC. Based on the results of the meta-analysis, it appears 


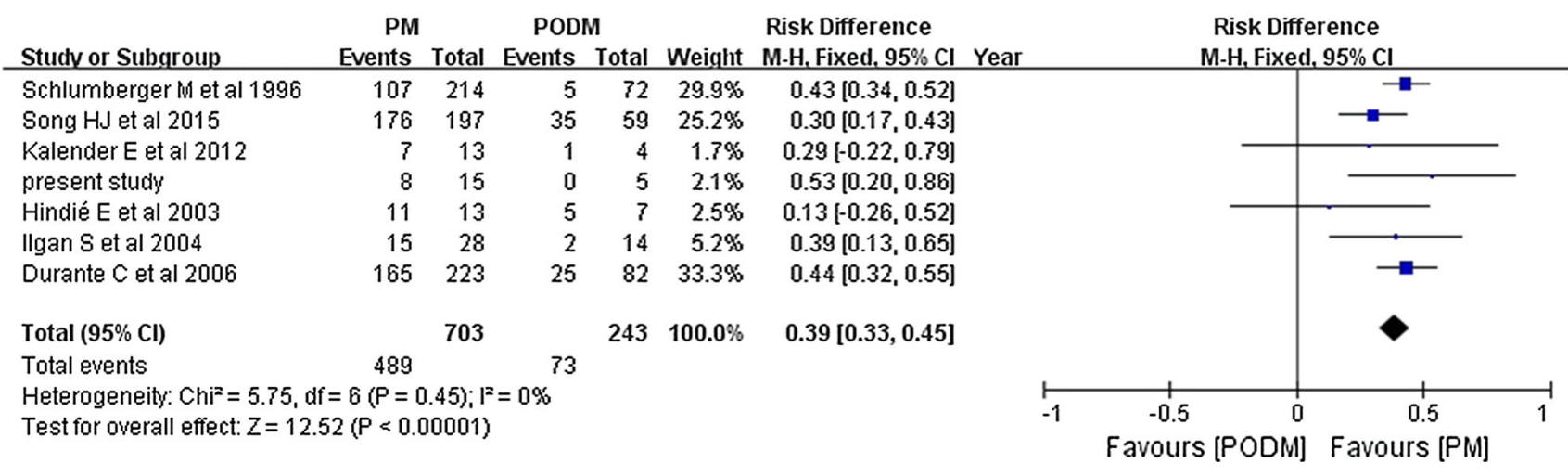

Fig. 3 Forest plots comparing the efficacy rates in the PM and PODM groups using a fixed-effects model. PM pulmonary metastasis, $P O D M$ pulmonary and other distant metastasis

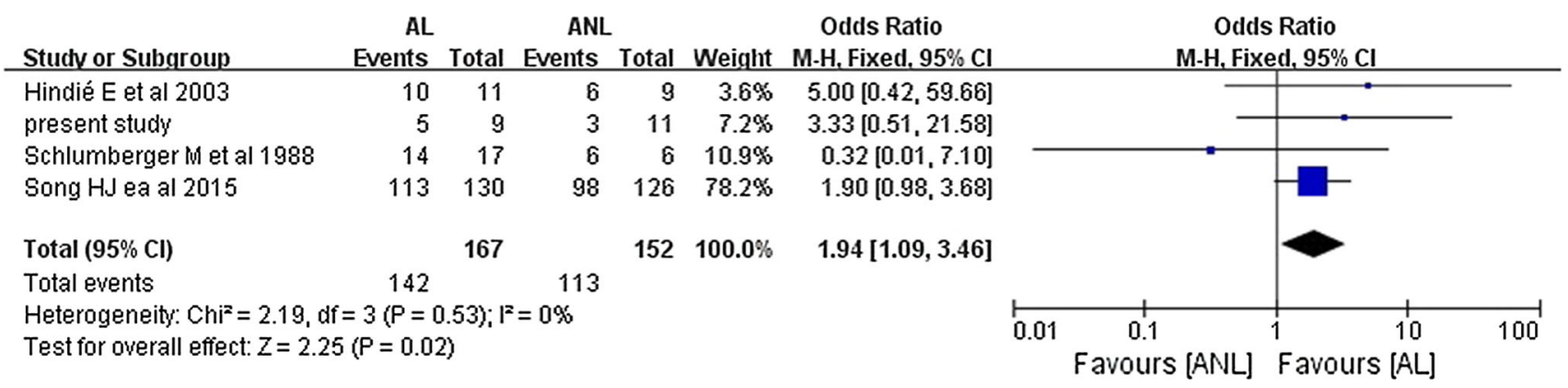

Fig. 4 Forest plots comparing the efficacy rates in the AL and ANL groups using a fixed-effects model. $A L$ age of $<40$ years, $A N L$ age of $\geq 40$ years

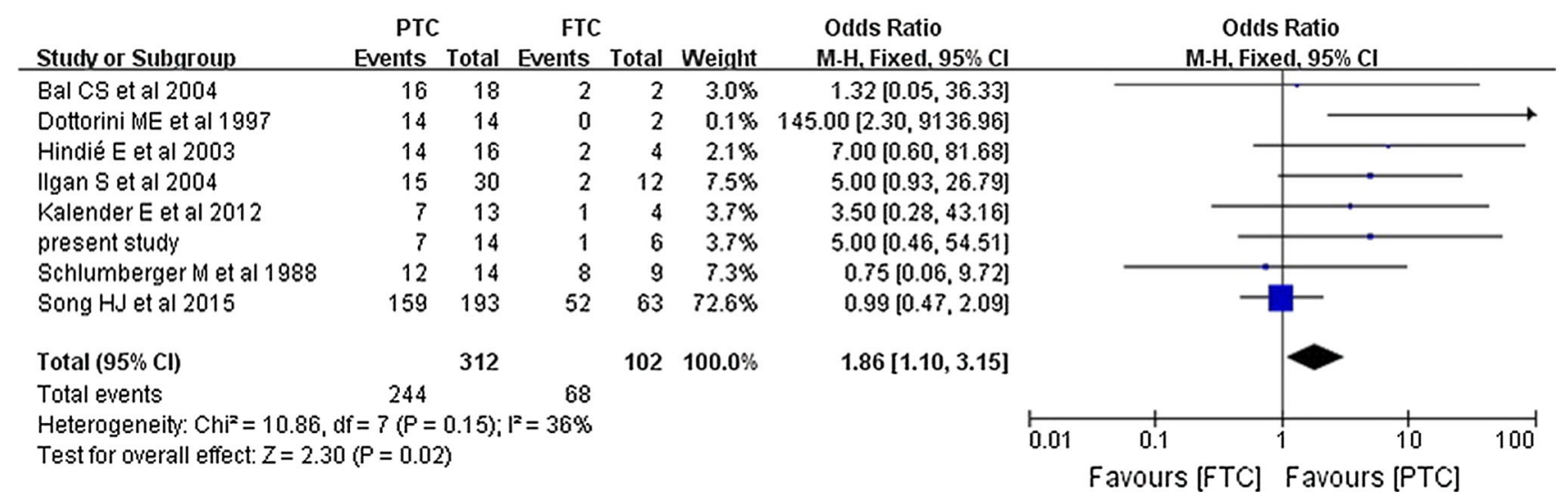

Fig. 5 Forest plots comparing the efficacy rates in the PTC and FTC groups using a fixed-effects model. PTC papillary thyroid cancer, FTC follicular thyroid cancer

that RAI efficacy is significantly predicted by the presence of PTC, an age of $<40$ years, the absence of non-lung distant metastasis and female patients. Further relevant studies needed to interpret differences above.

Similar to our results, some studies have revealed a low efficacy rate for RAI treatment of pulmonary metastasis from DTC [3, 12-16], although other studies have revealed higher efficacy rates [6-9, 17-23]. These differences may be related to the different criteria for dosage selection and efficacy evaluation. Secondly, the small sample size in the present study may be responsible for our inability to identify significant predictors of RAI efficacy. Lastly, the RAI efficacy is associated with the expression of NIS. The impairment of iodine uptake correlated directly with the RAI efficacy of DTC [10].

Older patients at DTC diagnosis often present with more aggressive cancer and have a decreased age adjusted disease-free and thus have worse prognosis [24, 25]. It is 


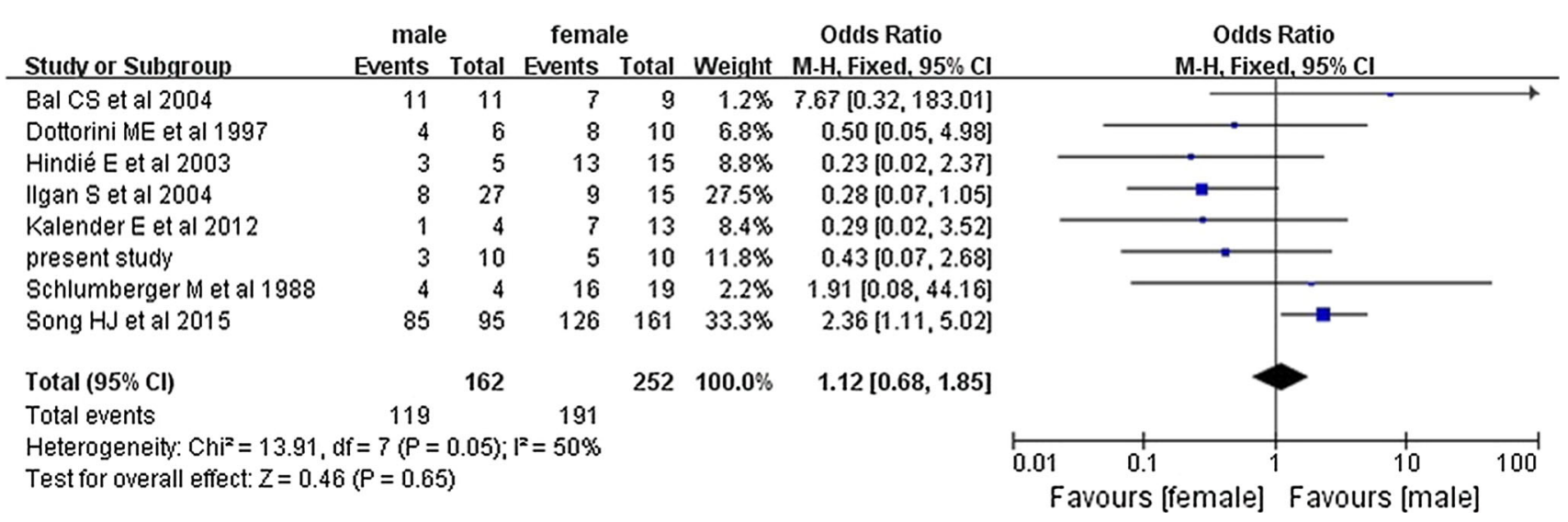

Fig. 6 Forest plots comparing the efficacy rates among men and women using a fixed-effects model

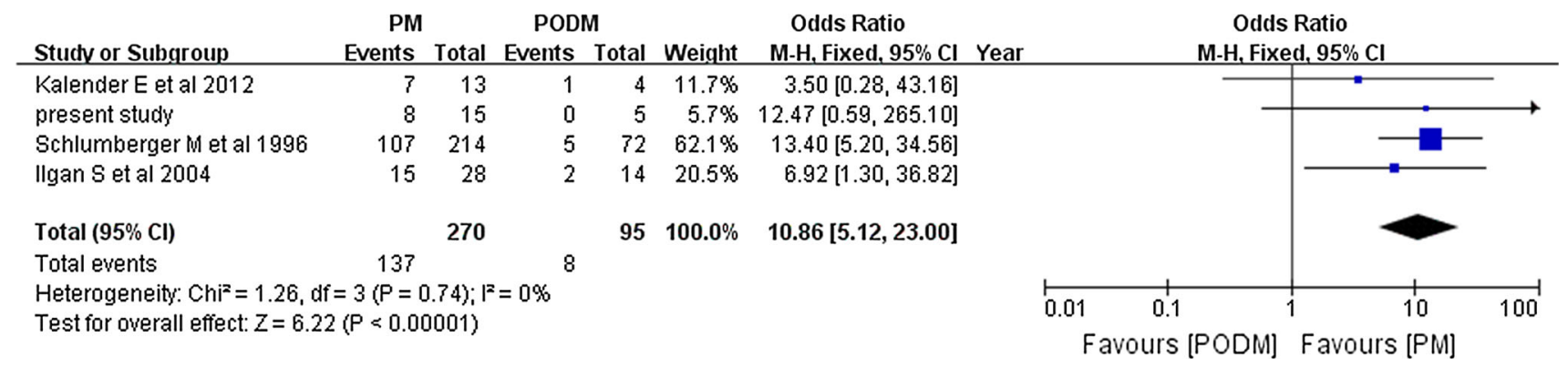

Fig. 7 Forest plots comparing the efficacy rates which correlated most closely with ours in the PM and PODM groups using a fixed-effects model. $P M$ pulmonary metastasis, $P O D M$ pulmonary and other distant metastasis

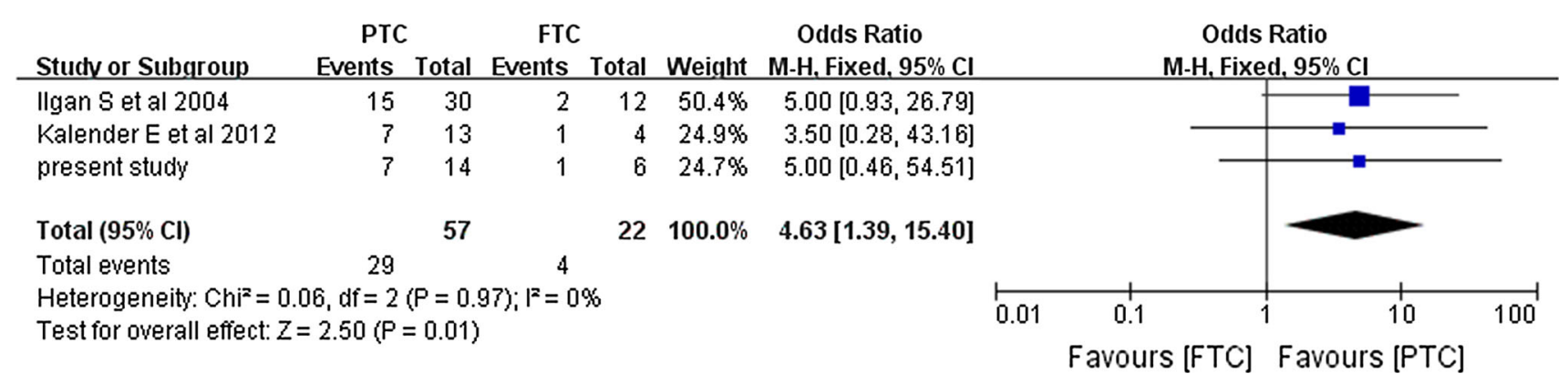

Fig. 8 Forest plots comparing the efficacy rates which correlated most closely with ours in the PTC and FTC groups using a fixed-effects model. PTC papillary thyroid cancer, FTC follicular thyroid cancer

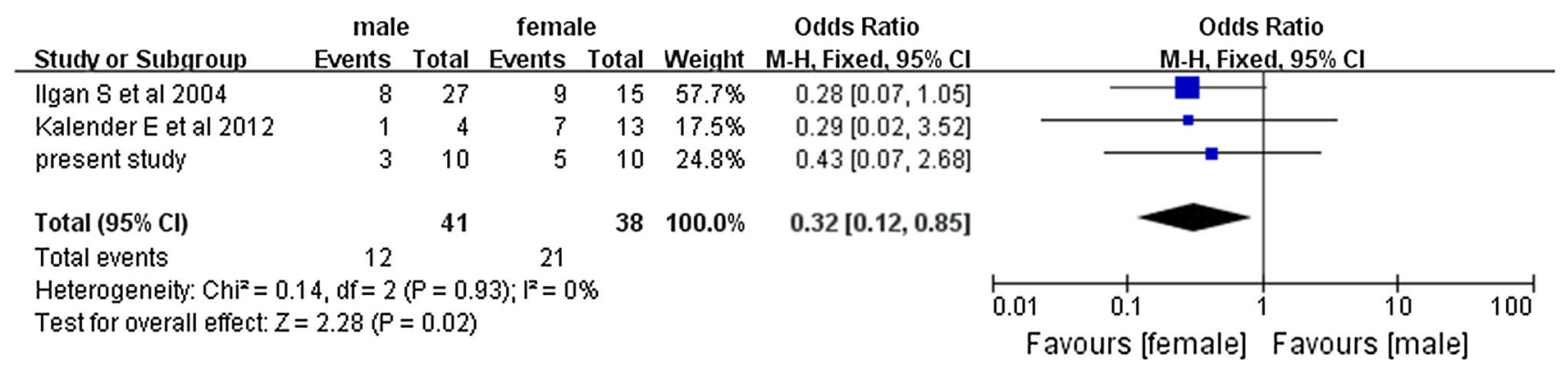

Fig. 9 Forest plots comparing the efficacy rates which correlated most closely to ours among men and women using a fixed-effects model 


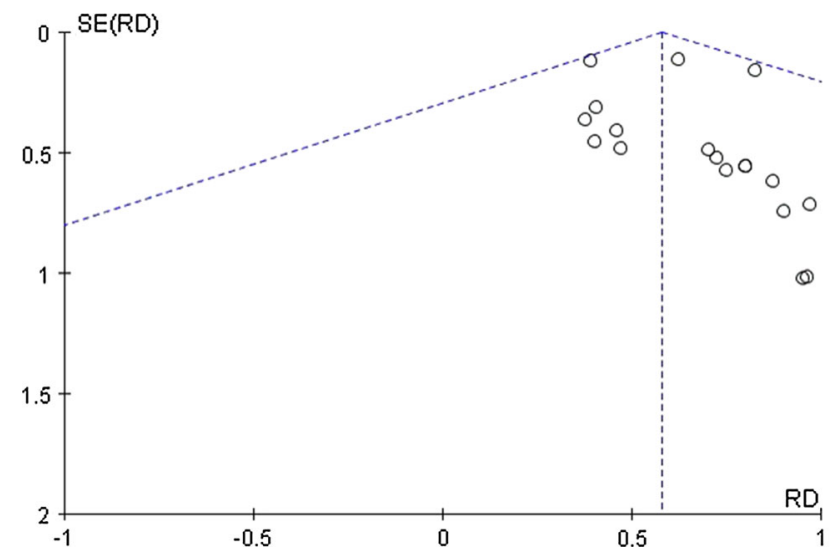

Fig. 10 Funnel plot for the included reports. $R D$ risk difference, $S E$ standard error

speculated that the difference of RAI efficacy between PTC and FTC is associated with the diverse metastatic gene signature for PTC and FTC [26]. Heterogeneity in the metastatic tissue may imply that iodine uptake may differ, which may be responsible for the significant difference of RAI efficacy between pulmonary metastasis patients and pulmonary and other distant metastasis patients [10]. As for gender, it is same to our first subgroup analysis about gender; most of the studies reported that there is no significant difference between male and female patients in RAI efficacy. But when we conducted a comparison between our retrospective study results and the results which correlated most closely with the results produced by us, we revealed the significant difference. Different criteria for evaluating the efficacy of RAI may be responsible for the failure to find the significant difference. We speculated that the difference may be associated with estrogen, but more large sample and multicenter clinical studies which have same criteria for evaluating the efficacy of RAI needed to confirm this new conclusion.

The present study has several limitations. First, the meta-analysis only included retrospective studies, and their findings may have been influenced by the absence of randomization or controlling for the limitations of ethical principles and medical reality. Second, each study used slightly different criteria for evaluating the efficacy of RAI. Finally, only the studies published in English were considered. Thus, after randomized controlled trials have been performed in this field, it may be appropriate to perform another meta-analysis to validate our findings.

RAI can be selected as a palliative treatment or a preoperative procedure for patients with pulmonary and bone metastasis while the radical surgical extirpation (complete bone metastasis surgery) could improve the survival for those patients [27, 28]. External radiotherapy is recommended for bone metastasis which cannot be readily excised surgically [29, 30]. Some patient groups with worse prognosis for the failure to uptake iodine may have a try to molecularly targeted therapy, which can increase NIS expression and radioiodine uptake in cancer cells according to some studies [31-33].

\section{Conclusions}

The present study revealed that RAI was an effective treatment for patients with pulmonary metastasis from DTC after surgical treatment. The efficacy of RAI was significantly predicted by the presence of PTC, age of $<40$ years, the absence of non-lung distant metastasis and female patients.

\section{Compliance with ethical standards}

Conflict of interest No conflict of interest exits regarding the submission of this manuscript.

Research involving human All the patients in this retrospective study came from the Department of Nuclear Medicine, the 88th PLA Hospital. The study complied with ethical standards and informed consent statement is not necessary for retrospective study.

Open Access This article is distributed under the terms of the Creative Commons Attribution 4.0 International License (http://crea tivecommons.org/licenses/by/4.0/), which permits unrestricted use, distribution, and reproduction in any medium, provided you give appropriate credit to the original author(s) and the source, provide a link to the Creative Commons license, and indicate if changes were made.

\section{References}

1. Sherman SI. Thyroid carcinoma. Lancet. 2003;361(9356):501-11.

2. Mazzaferri EL, Massoll N. Management of papillary and follicular (differentiated) thyroid cancer: new paradigms using recombinant human thyrotropin. Endocr Relat Cancer. 2002;9(4):227-47.

3. Durante C, Haddy N, Baudin E, Leboulleux S, Hartl D, Travagli JP, Caillou B, Ricard M, Lumbroso JD, De Vathaire F, et al. Long-term outcome of 444 patients with distant metastases from papillary and follicular thyroid carcinoma: benefits and limits of radioiodine therapy. J Clin Endocrinol Metab. 2006;91(8):2892-9.

4. Lin JD, Chao TC, Chou SC, Hsueh C. Papillary thyroid carcinomas with lung metastases. Thyroid. 2004;14(12):1091-6.

5. American Thyroid Association Guidelines Taskforce on Thyroid N, Differentiated Thyroid C, Cooper DS, Doherty GM, Haugen BR, Kloos RT, Lee SL, Mandel SJ, Mazzaferri EL, McIver B, et al. Revised American Thyroid Association management guidelines for patients with thyroid nodules and differentiated thyroid cancer. Thyroid. 2009;19(11):1167-214.

6. Bal CS, Kumar A, Chandra P, Dwivedi SN, Mukhopadhyaya S. Is chest X-ray or high-resolution computed tomography scan of the chest sufficient investigation to detect pulmonary metastasis in pediatric differentiated thyroid cancer? Thyroid. 2004;14(3):217-25.

7. Long B, Yang M, Yang Z, Yi H, Li L. Assessment of radioiodine therapy efficacy for treatment of differentiated thyroid cancer patients with pulmonary metastasis undetected by chest computed tomography. Oncol Lett. 2016;11(2):965-8.

8. Samuel AM, Rajashekharrao B, Shah DH. Pulmonary metastases in children and adolescents with well-differentiated thyroid cancer. J Nucl Med. 1998;39(9):1531-6.

9. Tachi Y, Iwano S, Kato K, Tadokoro M, Naganawa S. Diagnostic whole-body scanning before radioiodine therapy for pulmonary metastases of differentiated thyroid cancer: predictive value and recommendations. Clin Nucl Med. 2008;33(12):845-51. 
10. Chung JK, Youn HW, Kang JH, Lee HY, Kang KW. Sodium iodide symporter and the radioiodine treatment of thyroid carcinoma. Nucl Med Mol Imaging. 2010;44(1):4-14.

11. Higgins JP, Thompson SG, Deeks JJ, Altman DG. Measuring inconsistency in meta-analyses. BMJ. 2003;327(7414):557-60.

12. Kalender E, Zeki Celen Y, Elboga U, Deniz Demir H, Yilmaz M. Lung metastases in patients with differentiated thyroid carcinoma and evaluation of response to radioiodine therapy. Revista espanola de medicina nuclear e imagen molecular. 2012;31(6):328-31.

13. Ilgan S, Karacalioglu AO, Pabuscu Y, Atac GK, Arslan N, Ozturk E, Gunalp B, Ozguven MA. Iodine-131 treatment and high-resolution CT: results in patients with lung metastases from differentiated thyroid carcinoma. Eur J Nucl Med Mol Imaging. 2004;31(6):825-30.

14. Kucuk ON, Gultekin SS, Aras G, Ibis E. Radioiodine whole-body scans, thyroglobulin levels, 99mTc-MIBI scans and computed tomography: results in patients with lung metastases from differentiated thyroid cancer. Nucl Med Commun. 2006;27(3):261-6.

15. Pitoia F, Bueno F, Cross G. Long-term survival and low effective cumulative radioiodine doses to achieve remission in patients with 131Iodine-avid lung metastasis from differentiated thyroid cancer. Clin Nucl Med. 2014;39(9):784-90

16. Schlumberger M, Challeton C, De Vathaire F, Travagli JP, Gardet P, Lumbroso JD, Francese C, Fontaine F, Ricard M, Parmentier C. Radioactive iodine treatment and external radiotherapy for lung and bone metastases from thyroid carcinoma. J Nucl Med. 1996;37(4):598-605.

17. Biko J, Reiners C, Kreissl MC, Verburg FA, Demidchik Y, Drozd V. Favourable course of disease after incomplete remission on (131)I therapy in children with pulmonary metastases of papillary thyroid carcinoma: 10 years follow-up. Eur J Nucl Med Mol Imaging. 2011;38(4):651-5.

18. Hindie E, Melliere D, Lange F, Hallaj I, de Labriolle-Vaylet C, Jeanguillaume C, Lange J, Perlemuter L, Askienazy S. Functioning pulmonary metastases of thyroid cancer: does radioiodine influence the prognosis? Eur J Nucl Med Mol Imaging. 2003:30(7):974-81.

19. Lee YS, Lim YS, Lee JC, Wang SG, Kim IJ, Son SM, Lee BJ. Clinical implications of bilateral lateral cervical lymph node metastasis in papillary thyroid cancer: a risk factor for lung metastasis. Ann Surg Oncol. 2011;18(12):3486-92.

20. Pace L, Klain M, Albanese C, Salvatore B, Storto G, Soricelli A, Salvatore M Short-term outcome of differentiated thyroid cancer patients receiving a second iodine-131 therapy on the basis of a detectable serum thyroglobulin level after initial treatment. Eur J Nucl Med Mol Imaging. 2006;33(2):179-83.

21. Schlumberger M, Arcangioli O, Piekarski JD, Tubiana M, Parmentier C. Detection and treatment of lung metastases of differentiated thyroid carcinoma in patients with normal chest X-rays. J Nucl Med. 1988;29(11):1790-4.

22. Song HJ, Qiu ZL, Shen CT, Wei WJ, Luo QY. Pulmonary metastases in differentiated thyroid cancer: efficacy of radioiodine therapy and prognostic factors. Eur J Endocrinol. 2015;173(3):399-408.

23. Dottorini ME, Vignati A, Mazzucchelli L, Lomuscio G, Colombo L. Differentiated thyroid carcinoma in children and adolescents: a 37-year experience in 85 patients. J Nucl Med. 1997;38(5):669-75.

24. Eustatia-Rutten CF, Corssmit EP, Biermasz NR, Pereira AM, Romijn JA, Smit JW. Survival and death causes in differentiated thyroid carcinoma. J Clin Endocrinol Metab. 2006;91(1):313-9.

25. Ross DS. Long-term management of differentiated thyroid cancer. Endocrinol Metab Clin N Am. 1990;19(3):719-39.

26. Nixon IJ, Whitcher MM, Palmer FL, Tuttle RM, Shaha AR, Shah JP, Patel SG, Ganly I. The impact of distant metastases at presentation on prognosis in patients with differentiated carcinoma of the thyroid gland. Thyroid. 2012;22(9):884-9.

27. Bernier MO, Leenhardt L, Hoang C, Aurengo A, Mary JY, Menegaux F, Enkaoua E, Turpin G, Chiras J, Saillant G, et al. Survival and therapeutic modalities in patients with bone metastases of differentiated thyroid carcinomas. J Clin Endocrinol Metab. 2001;86(4):1568-73.

28. Bonichon F, Buy X, Godbert Y, Pointillart V, Henriques de Figueiredo B, Gangi A, Palussiere J. Local treatment of metastases from differentiated thyroid cancer. Annales d'endocrinologie. 2015;76(1 Suppl 1):1S40-6.

29. Tubiana M, Lacour J, Monnier JP, Bergiron C, Gerard-Marchant R, Roujeau J, Bok B, Parmentier C. External radiotherapy and radioiodine in the treatment of 359 thyroid cancers. Br J Radiol. 1975;48(575):894-907.

30. Tubiana M, Haddad E, Schlumberger M, Hill C, Rougier P, Sarrazin D. External radiotherapy in thyroid cancers. Cancer. 1985;55(9 Suppl):2062-71.

31. Schmutzler C. Regulation of the sodium/iodide symporter by retinoids-a review. Exp Clin Endocrinol Diabetes. 2001;109(1):41-4.

32. Liu YY, Stokkel MP, Pereira AM, Corssmit EP, Morreau HA, Romijn JA, Smit JW. Bexarotene increases uptake of radioiodide in metastases of differentiated thyroid carcinoma. Eur J Endocrinol. 2006;154(4):525-31.

33. Ho AL, Grewal RK, Leboeuf R, Sherman EJ, Pfister DG, Deandreis D, Pentlow KS, Zanzonico PB, Haque S, Gavane S, et al. Selumetinib-enhanced radioiodine uptake in advanced thyroid cancer. N Engl J Med. 2013;368(7):623-32. 